\title{
Guarding against emerging epidemics: addressing HIV and AIDS among culturally and linguistically diverse communities in NSW
}

\author{
Tadgh McMahon ${ }^{\mathrm{A}, \mathrm{B}}$, Renee J. Moreton ${ }^{\mathrm{A}}$ \\ and Barbara N. Luisi ${ }^{\mathrm{A}}$ \\ ${ }^{\mathrm{A}}$ Multicultural HIV/AIDS and Hepatitis C Service, \\ Sydney South West Area Health Service \\ ${ }^{\mathrm{B} C o r r e s p o n d i n g ~ a u t h o r . E m a i l: ~ m c m a h t @ e m a i l . c s . n s w . g o v . a u ~}$
}

\begin{abstract}
In Australia, people from culturally and linguistically diverse backgrounds, largely born in low- and middle-income countries, accounted for $24 \%$ of HIV notifications in the period 2004-2008. NSW has one of the most culturally diverse communities in Australia and is also the most popular destination for both permanent and temporary migrants. Consequently people from culturally and linguistically diverse backgrounds have recently been recognised as a priority in the NSW HIV/AIDS Strategy. The National Health and Medical Research Council provides a cultural competency framework for re-orienting public health and health promotion programs to better meet the needs of these communities. It is being applied to re-orientate the HIV response in NSW. Examples of how this framework can be implemented are provided.
\end{abstract}

New South Wales (NSW) has one of the most culturally diverse communities in Australia, and people from culturally and linguistically diverse (CALD) backgrounds have recently been recognised as a priority in the NSW HIV/ AIDS Strategy. This diversity presents challenges and opportunities for the human immunodeficiency (HIV) and acquired immunodeficiency syndrome (AIDS) sector in re-orienting health promotion, treatment and care services to better reach CALD communities in NSW. This paper will outline how cultural competency provides a framework for the re-orientation of the HIV response in NSW and provides specific examples of how this framework can be implemented.

Global population mobility and accelerating international migration to high-income countries ${ }^{1}$ has transformed the demography of most industrialised countries over the past 50 years. ${ }^{2}$ Population mobility and migration are historically some of the forces associated with the emergence and spread of infectious diseases by bringing populations with disparate prevalence rates into closer proximity with each other. ${ }^{3}$ This disparity is acute in the global incidence of HIV with more than $95 \%$ of annual HIV infections occurring in low- and middle-income countries. ${ }^{4}$ Migrants and mobile populations, especially those from low- and middle-income countries, may also have specific social vulnerabilities to HIV including social isolation, poverty, limited access to health services and information - all of which can be compounded by cultural and linguistic barriers in the destination country. ${ }^{5}$

\section{Migration to Australia}

Australia's annual migrant intake of about 150000 permanent settlers is mostly made up of skilled and family migration with humanitarian entrants accounting for about 10000 settlers. $^{6}$ Since the mid 1980s all applicants for permanent migration to Australia undergo HIV testing as part of the health requirements. ${ }^{7}$ In addition, temporary migration, mostly made up of students and temporary skilled entrants, is on the rise, with NSW the most popular destination for both permanent settlers and temporary entrants. ${ }^{6}$

\section{HIV among people from CALD backgrounds}

In Australia, people from CALD backgrounds, largely born in low- and middle-income countries, accounted for $24 \%$ of HIV notifications in the period 2004-2008. ${ }^{8}$ In 2008, people born in Asia and Sub-Saharan Africa made up $21 \%$ of all HIV notifications, with the highest agestandardised incidence rate among people born in SubSaharan Africa. ${ }^{8}$ This pattern of HIV among immigrants in Australia is broadly in line with trends seen in the European Union, ${ }^{9}$ the USA, ${ }^{10,11}$ and Canada. ${ }^{12}$

The dominant modes of HIV transmission among people from CALD backgrounds in NSW tend to mirror epidemics in their regions of birth, with higher proportions of heterosexual transmission of HIV, and consequently higher proportions of women with HIV. ${ }^{13}$ Information describing 'Country of birth' and 'language spoken at home' has been collected with HIV notifications in NSW since the mid1990 s with reliable data available since $2000 .^{13}$ An unpublished analysis of NSW HIV surveillance data from 
2000-2007 indicated that homosexual and heterosexual transmission each account for about half of HIV notifications in NSW among people from CALD backgrounds. ${ }^{13}$ Overall, people from CALD backgrounds accounted for $53.4 \%$ of heterosexual transmission and $15.3 \%$ of homosexual transmission in NSW from 2000-2007. ${ }^{13}$ The analysis also found that, while HIV is spread across multiple CALD communities, notifications are predominantly drawn from low- and middle-income countries in Asia and Sub-Saharan Africa, with these two regions accounting for $15 \%$ of overall notifications from 2000-2007. ${ }^{13}$ The priority CALD populations for HIV health promotion, treatment and care in NSW are predominantly drawn from these two regions.

Epidemiological and social research points to underlying differences in the social and cultural dimensions of HIV for people from CALD backgrounds in NSW. Epidemiological data indicate higher rates of late presentation with HIV. ${ }^{8}$ Rates of HIV testing have been found to be much lower in surveys of Asian gay men in Sydney. ${ }^{14,15}$ Surveys among wider CALD communities point to high levels of HIV knowledge and awareness but variable practice to prevent HIV and limited use of health services despite being eligible for Medicare. ${ }^{16}$ Living with HIV also has a different impact for people from CALD backgrounds. Research indicates that they are often faced with negotiating two major life disruptions simultaneously: an HIV diagnosis and the stressors of migration. ${ }^{7}$ These underlying differences underscore the need for a targeted and tailored response to HIV in CALD communities.

\section{Emerging challenges}

There is an increasing trend of temporary migration to Australia alongside a largely stable permanent immigration intake. Over the past 5 years, temporary migration accounted for an annual average of more than 600000 people residing temporarily in Australia, dwarfing the permanent immigration intake. ${ }^{6}$ Temporary entrants, mostly students and skilled workers, typically reside in Australia for up to 4 years at a time. In addition to the large numbers of temporary migrants, the mobility of the general population is unprecedented today in volume, speed and reach, ${ }^{17}$ focussing attention on travel and mobility, particularly to and from high HIV prevalence countries, as a potential driver of HIV transmission.

These factors combine to raise important public health practice and policy challenges in NSW, in particular, how to identify and engage in HIV health promotion with large, long-term transient populations. Immigrant health policy in this context of increasing temporary migration and population mobility should be guided by a greater recognition of the dynamics of population migration and mobility, background prevalence rates of HIV between destination and source countries, and implementing measured strategies to manage threats based on actual and significant risks to public health. ${ }^{18}$

\section{Addressing challenges through a cultural competency framework}

The National Health and Medical Research Council (NHMRC) provides a cultural competency framework for re-orienting public health and health promotion programs to better meet the needs of CALD communities. ${ }^{19}$ The four dimensions of the framework - systemic, organisational, professional and individual - interrelate 'so that cultural competence at an individual and professional level is underpinned by systemic and organisational commitment and capacity'. ${ }^{19}$ Cultural competence within the health system is more than awareness of cultural differences and is best viewed as a developmental process for individual workers, the agency where they work and the health system in which they work. ${ }^{20}$

\section{Systemic approaches}

The HIV response in NSW has been well served by targeted primary and secondary interventions with the most affected communities; an approach that can also be employed with priority CALD communities. A systemic culturally competent approach along the lines proposed by the NHMRC would continue to work towards ensuring that we can capture, enumerate and measure diversity in surveillance to inform planning and prioritising programs for CALD communities in NSW. ${ }^{19}$ This systemic approach to HIV prevention is central to the identification of priority populations. An example of this approach undertaken in NSW has been targeted work with Thai-born gay men to increase regular testing for HIV and other sexually transmitted infections. A partnership of HIV-sector stakeholders planned and implemented a social marketing campaign which was placed in both the Thai and gay media to maximise its reach and coincided with an annual Thai cultural milestone to maximise its salience.

\section{Organisational approaches}

An organisational approach requires agencies to reflect on and review current practices, policies and planning as a step towards delivering more culturally competent programs and services. The need for an organisational approach was highlighted in an HIV-sector needs analysis commissioned by the Multicultural HIV/AIDS and Hepatitis C Service in $2007 .^{20}$ It found that HIV agencies perceived they lacked the knowledge, skills and self-efficacy to work with CALD communities. $^{20}$ A cultural competence training program subsequently developed by the Multicultural HIV/AIDS and Hepatitis C Service for the HIV sector focused on the development of an agency-level response to CALD populations. An evaluation of the training indicated that this model was effective in supporting organisations to identify and commit resources to future strategies to deliver a sustained response with priority CALD populations. ${ }^{20}$ 


\section{Professional and individual approaches}

These approaches build the skills of individuals and professionals to work with cultural diversity, including understanding cultural dynamics and enhancing communication skills. Skills developed at this level build upon, and are supported by, systemic and organisational approaches. ${ }^{19}$ An example of this approach in NSW was recent work undertaken by the NSW CALD HIV Interagency which identified HIV and tuberculosis co-infection as a clinical priority affecting CALD populations. A revised HIV and tuberculosis policy directive was the systemic change that provided the impetus for workforce development initiatives with HIV, sexual health and tuberculosis services to enhance screening and improve the management of HIV and tuberculosis in CALD patients.

\section{Conclusion}

Guarding against the emerging epidemic of HIV in CALD communities will require proportionate and culturallycompetent activity across the spectrum of the public health response to HIV in NSW in health promotion, treatment and care. This spectrum of activity should continue to refine HIV policy frameworks; build more detailed epidemiological profiles of homosexual and heterosexual transmission of HIV in CALD communities to better identify priority populations; and research the social contexts and understandings of HIV and AIDS in these populations. At the same time, efforts to enhance the cultural competence of HIV sector agencies and their staff will support the building of relationships, trust and partnerships with the most affected CALD communities to ensure that HIV interventions are culturally appropriate and less likely to stigmatise people with, or at risk of, HIV and AIDS.

\section{References}

1. The World Bank. Country Groups. Available from: http://web worldbank.org (Cited 22 December 2009.)

2. Castles S. Ethnicity and globalisation. London: Sage; 2000.

3. Apostolopoulos Y, Sonmez S. Demographic and epidemiological perspectives of human movement. In: Apostolopoulos Y, Sonmez S, editors. Population Mobility and Infectious Disease. New York: Springer; 2007. pp. 3-20.

4. UNAIDS. AIDS Outlook 2009: World AIDS Day Report 2008. Geneva: UNAIDS; 2008. Available from: http://www.unaids. org (Cited 15 August 2009.)

5. UNAIDS. Population mobility and AIDS. Geneva: UNAIDS; 2001. Available from: http://www.unaids.org (Cited 20 February 2009.)

6. Department of Immigration and Citizenship (DIAC). Immigration Update 2008-2009. Canberra: DIAC; 2009. Available from: http://www.immi.gov.au/media/publications/statistics (Cited 15 November 2009.)
7. Korner H, Petrohilos M, Madeddu D. Living with HIV and cultural diversity in Sydney. Sydney: National Centre in HIV Social Research, University of NSW; 2005.

8. National Centre in HIV Epidemiology and Clinical Research. HIV/AIDS, viral hepatitis and sexually transmissible infections in Australia Annual Surveillance Report 2009. National Centre in HIV Epidemiology and Clinical Research, The University of New South Wales, Sydney, NSW, 2009. Available at: http://www.nchecr.unsw.edu.au/ NCHECRweb.nsf/page/Annual+Surveillance+Reports (Cited 16 September 2009.)

9. del Amo J, Broring G, Hamers F, Infuso A, Fenton KA. Monitoring HIV/AIDS in Europe's migrant communities and ethnic minorities. AIDS 2004; 18: 1867-73. doi:10.1097/ 00002030-200409240-00002

10. Chin JJ, Leung M, Sheth L, Rodriquez TR. Let's not ignore a growing problem for Asians and Pacific Islanders in the US. $J$ Urban Health 2007; 84(5): 642-7. doi:10.1007/ s11524-007-9200-8

11. Herbst JH, Kay LS, Passin WF, Lyles CM, Crepaz N, Marin BV. A systematic review and meta-analysis of behavioral interventions to reduce HIV risk behaviors of Hispanics in the United States and Puerto Rico. AIDS Behav 2007; 11(1): 25-47. doi:10.1007/s10461-006-9151-1

12. Boulos D, Yan P, Schnader D, Remis R, Archibald C. Estimates of HIV prevalence and incidence in Canada 2005. Can Commun Dis Rep 2006; 32(15): 165-74.

13. Ward K, Micallef J. Analysis of NSW HIV notifications among culturally and linguistically diverse (CALD) communities 2000-2007 (unpublished data). Sydney: Communicable Diseases Branch, NSW Department of Health; 2008.

14. Mao L, Van de Ven P, Wang J, Hua M, Prihaswan P, Ku A. Asian Gay Community Periodic Survey. Sydney: National Centre in HIV Social Research, University of NSW; 2003.

15. Prestage G, Van de Ven P, Wong K, Mahat M, McMahon T. Asian Gay Men in Sydney. Sydney: National Centre in HIV Social Research, University of NSW; 2001.

16. Asante A, Korner H, McMahon T, Sabri W, Kippax S. Periodic survey of HIV knowledge and use of health services among people from CALD backgrounds, 2006-2008. Sydney: National Centre in HIV Social Research, University of NSW; 2009. Available from: http://nchsr.arts.unsw.edu.au (Cited 2 August 2009.)

17. Wilson ME. Population mobility and the geography of microbial threats. In: Apostolopoulos Y, Sonmez S, editors. Population Mobility and Infectious Disease. London: Springer; 2007. pp. 21-39.

18. Gushulak BD, MacPherson DW. Migration in a mobile world: health, population mobility, and emerging disease. In: Apostolopoulos Y, Sonmez S, editors. Population Mobility and Infectious Disease. New York: Springer; 2007. pp. 283-300.

19. National Health and Medical Research Council (NHMRC). Cultural competency in health: a guide for policy, partnerships and participation. Canberra: NHMRC; 2006.

20. Eisenberg M, Moreton R, McMahon T, Ray N. HIV, diversity and cultural competence. Social Research Brief 12. Sydney: National Centre in HIV Social Research, University of NSW; 2009. Available from: http://nchsr.arts.unsw.edu.au (Cited 12 September 2009.) 